\title{
Synthetic MRI of the brain in a clinical setting
}

Ida Blystad, Jan Bertus Marcel Warntjes, Örjan Smedby, Anne-Marie Landtblom, Peter

Lundberg and Elna-Marie Larsson

Journal Article

\section{Tweet}

N.B.: When citing this work, cite the original article.

Original Publication:

Ida Blystad, Jan Bertus Marcel Warntjes, Örjan Smedby, Anne-Marie Landtblom, Peter Lundberg and Elna-Marie Larsson. Synthetic MRI of the brain in a clinical setting, Acta Radiologica, 2012. 53(10), pp. 1158-1163.

http://dx.doi.org/10.1258/ar.2012.120195

Copyright: SAGE Publications (UK and US)

http://www.uk.sagepub.com/home.nav

Postprint available at: Linköping University Electronic Press

http://urn.kb.se/resolve?urn=urn:nbn:se:liu:diva-89641 


\title{
Synthetic Mri of the Brain in a Clinical Setting
}

\author{
I Blystad, JBM Warntjes, O Smedby, A-M Landtblom, \\ P Lundberg and E-M Larsson
}

\begin{abstract}
Background: Conventional magnetic resonance imaging (MRI) has relatively long scan times for routine examinations, and the signal intensity of the images is related to the specific MR scanner settings. Due to scanner imperfections and automatic optimizations, it is impossible to compare images in terms of absolute image intensity. Synthetic MRI, a method to generate conventional images based on MR quantification, potentially both decreases examination time and enables quantitative measurements.

Purpose: To evaluate synthetic MRI of the brain in a clinical setting by assessment of the contrast, the contrast-to-noise ratio (CNR), and the diagnostic quality compared with conventional MR images.

Material and Methods: Twenty-two patients had synthetic imaging added to their clinical MR examination. In each patient, 12 regions of interest were placed in the brain images to measure contrast and CNR. Furthermore, general image quality, probable diagnosis, and lesion conspicuity were investigated.

Results: Synthetic T1-weighted spin echo and T2-weighted turbo spin echo images had higher contrast but also a higher level of noise, resulting in a similar CNR compared with conventional images. Synthetic T2-weighted FLAIR images had lower contrast and a higher level of noise, which led to a lower CNR. Synthetic images were generally assessed to be of inferior image quality, but agreed with the clinical diagnosis to the same extent as the
\end{abstract}


conventional images. Lesion conspicuity was higher in the synthetic T1w images, which also had a better agreement with the clinical diagnoses than the conventional $\mathrm{T} 1 \mathrm{w}$ images.

Conclusion: Synthetic MR can potentially shorten the MR examination time. Even though the image quality is perceived to be inferior, synthetic images agreed with the clinical diagnosis to the same extent as the conventional images in this study.

Keywords: CNS, MR-Imaging, Brain, Technology Assessment, Imaging sequences

Magnetic resonance imaging (MRI) provides high soft tissue contrast, and hence a high sensitivity for detecting pathological changes in the brain. Despite development of faster imaging techniques, the scan time for routine MRI of the brain is rather long, normally 30-45 minutes for a routine clinical examination. The exam consists of sequentially acquired pulse sequences and each sequence has different image characteristics depending on the scanner settings. In addition, other parameters, such as the inhomogeneity of the radiofrequency (RF) field $B_{1}$ and the coil sensitivity profile affect the image appearance. Furthermore, the RF chain amplification settings are re-optimized for each patient and geometry. As a result the signal intensity in the images will unavoidably vary from one examination to the next, making it impossible to directly compare exams in terms of absolute signal intensity values, leaving only the signal differences, the contrast, as useful information. This problem can be solved by absolute quantification of the physical properties of the tissue, e.g. the longitudinal $\mathrm{R}_{1}$ relaxation rate, the transverse $\mathrm{R}_{2}$ relaxation rate, and the proton density PD. These physical properties are independent of the scanner settings and imperfections and provide a quantitative basis for individual patient follow-up and for comparison between patients. A quantitative MRI sequence can be used to measure $\mathrm{R}_{1}, \mathrm{R}_{2}$, and $\mathrm{PD}$, and from these parameters it is possible to calculate the expected pixel intensity for conventional MR images with a free choice of echo time (TE), repetition time (TR), and inversion delay time (TI) (1-5). This approach is called synthetic MRI and is used to create T1- and T2-weighted (w) images for 
qualitative clinical MRI evaluation. Synthetic MRI is potentially faster than conventional MRI scanning if many different contrast settings are required for the same geometry, which is usually the case in a clinical setting. A shorter scan time is an advantage when examining pediatric patients or non-cooperative patients e.g. with dementia. Previous quantitative MRI sequences have not been clinically applicable due to long acquisition times (6) or long postprocessing times (7). An advantage of a quantitative sequence is the possibility of consistent image comparison and automatic tissue assessment (8-11), as pointed out already in the original work on MRI by Lauterbur (12). Absolute quantification of the physical properties of the tissue could be useful for lesion characterization and for the evaluation of therapeutic response. A prerequisite for the clinical application of synthetic MRI, however, is that the images have similar diagnostic quality as the corresponding conventional MR images.

In the present study, a new quantitative MRI method was assessed (13). Quantification was performed with a single MRI acquisition with less than 6 minutes scan time to measure the absolute values of $R_{1}, R_{2}$, and $P D$. The aim of the study was to evaluate this method of synthetic MRI of the brain in a clinical setting by assessing the contrast, the contrast-to-noise ratio (CNR), and the diagnostic quality compared with conventional MR images.

\section{Material and Methods}

\section{Patients}

Twenty-two patients (mean age 53 years, range 21-85) referred to the MR unit from the department of neurology were asked by their neurologist to participate, and if they agreed, the new quantitative MRI sequence was added to their clinical MRI scan of the brain. Eight patients had known multiple sclerosis (MS), seven patients had experienced an ischemic event, and seven patients had an unclear diagnosis. The local ethics committee approved the 
study protocol, and informed written consent was obtained from all patients before entering the study.

\section{MR sequences}

Patients were examined according to a clinical protocol, standard brain, or MS protocol, with the addition of the new quantitative sequence. Conventional T1-w spin echo images, T2-w turbo spin echo images, and T2-w fluid attenuated inversion recovery (FLAIR) images were acquired in 3.39, 2.01, and $4.30 \mathrm{~min}$ (total scan time $10.10 \mathrm{~min}$, standard brain protocol) and 5.11, 3.42, and $6.01 \mathrm{~min}$, (total scan time $14.54 \mathrm{~min}$, MS protocol), respectively. The acquisition details are given in Table 1.

Table 1. Acquisition parameters for the T1-w, T2-w and FLAIR acquisitions of the standard (a) and MS (b) protocol: the echo time (TE), the repetition time (TR), the echo train length (ETL), the SENSE acceleration factor, the number of averages sampled (NSA), the number of slices, the slice thickness, the in-plane resolution and the acquisition scan time. The FLAIR sequences had an inversion time of 2000 ms. The QRAPMASTER (QM) acquisition parameters are added. All images are in the axial plane.

\begin{tabular}{|c|c|c|c|c|c|c|c|c|c|}
\hline & $\begin{array}{l}\text { TE } \\
(\mathrm{ms})\end{array}$ & $\begin{array}{c}\text { TR } \\
\text { (ms) }\end{array}$ & ETL & SENSE & NSA & Slices & $\begin{array}{c}\text { Thickness } \\
\text { (mm) }\end{array}$ & $\begin{array}{c}\text { Resolution } \\
\left(\mathrm{mm}^{2}\right)\end{array}$ & $\begin{array}{l}\text { Scan time } \\
\text { (minutes) }\end{array}$ \\
\hline $\mathrm{T} 1-\mathrm{w}^{\mathrm{a}}$ & 15 & 390 & 1 & 1.5 & 2 & 24 & 5.0 & $0.8 \times 1.0$ & $3: 39$ \\
\hline $\mathrm{T} 2-\mathrm{w}^{\mathrm{a}}$ & 100 & 4800 & 15 & 2.0 & 3 & 24 & 5.0 & $0.9 \times 1.1$ & $2: 01$ \\
\hline FLAIR $^{\mathrm{a}}$ & 120 & 6000 & 23 & 1.0 & 2 & 24 & 5.0 & $0.6 \times 0.8$ & $4: 30$ \\
\hline $\mathrm{T} 1-\mathrm{w}^{\mathrm{b}}$ & 15 & 390 & 1 & 1.3 & 2 & 44 & 3.0 & $0.9 \times 1.1$ & $5: 11$ \\
\hline$T 2-w^{b}$ & 100 & 4800 & 15 & 2.0 & 3 & 44 & 3.0 & $0.9 \times 1.1$ & $3: 42$ \\
\hline FLAIR $^{b}$ & 120 & 6000 & 23 & 1.0 & 2 & 44 & 3.0 & $0.6 \times 0.8$ & $6: 01$ \\
\hline QM & $6 * 15$ & 2700 & 6 & 2.0 & 1 & 24 & 6.0 & $1.0 \times 1.0$ & $5: 48$ \\
\hline
\end{tabular}


The quantitative MRI sequence, QRAPMASTER, (13) is a saturation recovery turbo spinecho sequence with four different saturation delays at 130, 380, 1230, and $2580 \mathrm{~ms}$. Each acquisition consisted of a multi-echo read-out with six echoes at multiples of $15 \mathrm{~ms}$. Further acquisition details are also given in Table 1 . This resulted in a matrix of 4 x $6=24$ images per slice with different saturation delay times and different echo times.

A least square fit was performed on the signal intensity I of these images according to:

$I=A \cdot P D \cdot \exp \left(-R_{2} \cdot T E\right) \cdot \frac{1-\left[1-\cos \left(B_{1} \theta\right)\right]-\exp \left(-R_{1} \cdot T I\right)-\cos \left(B_{1} \theta\right) \cdot \exp \left(-R_{1} \cdot T R\right)}{1-\cos \left(B_{1} \alpha\right) \cdot \cos \left(B_{1} \theta\right) \cdot \exp \left(-R_{1} \cdot T R\right)}$

to retrieve $R_{1}, R_{2}, P D$ and the normalized radio-frequency $B_{1}$ field. (13)The applied excitation flip angle $\alpha$ and the saturation pulse angle $\theta$ were obtained from the scanner, $A$ is an overall intensity scaling factor taking into account the coil sensitivity, the RF chain amplification, the voxel volume, etc. A 1.5T Achieva scanner was used (Philips, Best, the Netherlands). Synthetic images, matching the conventional scanner settings, were created using SyMRI Diagnostics (SyntheticMR AB, Linköping, Sweden). The $B_{1}$ field was set to 1 for these images. Post-processing time of the raw image dataset was less than 1 minute on an ordinary PC, after which synthetic images were generated in real time.

\section{Contrast and CNR measurements}

A neuroradiologist drew small circular regions of interest (ROIs) to assess the contrast and the CNR. The ROIs, approximately $4 \mathrm{~mm}$ in diameter, were positioned in cerebrospinal fluid (bilaterally in the anterior horns of the lateral ventricles), in grey matter (bilaterally in the 
thalamus, in the occipital cortex, and the frontal cortex), and in white matter (bilaterally in the centrum semiovale, and for the corpus callosum one in the genu and one in the splenium), in total 12 ROIs per patient. These regions were included to obtain representative values from the different tissue types without partial volume effect. White matter lesions were present in 15 patients, and a ROI was drawn in one such lesion in each of these patients. The mean value and the standard deviation of the signal intensity within each ROI were recorded. The contrast between ROIs was calculated as the intensity difference divided by the sum of the intensities, and the CNR was calculated as the intensity difference divided by the median standard deviation of all 12 ROIs in each patient.

\section{Visual evaluation}

Image sets were anonymized and transferred as DICOM files to a visual grading software package ViewDEX, University of Gothenburg, Sweden (14). The sequences were assessed separately in random order by two neuroradiologists at different times and blinded to each other. Image quality was graded according to the following eight criteria:

1. Differentiation of grey-white matter at the level of the lateral ventricles

2. Demarcation of caudate nucleus head at the level of foramina of Monro

3. Demarcation of the lentiform nucleus at the level of foramina of Monro

4. Demarcation of the sulci

5. Perceived level of signal to noise

6. Parallel imaging (SENSE) artifacts

7. Pulsation artifacts

8. Other artifacts (e.g. motion)

Criteria 1-4 were modified from applicable parts of the European guidelines for assessment on image quality (15). Criteria 5-8 concern more general image quality parameters. The 
following scoring scale was used: 1, Inadequate; 2, Sufficient; 3, Good; 4, Excellent. For criteria 6-8, "excellent" was interpreted as the complete absence of artifacts.

Finally, the observers were asked to make a probable diagnosis (MS, ischemia, or nonspecific) based upon the blinded evaluation of each image sequence. The clinical diagnosis was regarded as the correct diagnosis.

After the individual blinded assessment of the image sets, an additional evaluation was performed with the corresponding conventional and synthetic images placed adjacent to each other. A consensus qualitative evaluation was done by the two neuroradiologists to determine whether the conventional or the synthetic images were superior for the visualization of white matter lesions.

\section{Statistical Analysis}

Correlation between the contrast and CNR measurements of synthetic and conventional images was analyzed using linear regression. Visual scores for each of the image quality criteria 1-8 were analyzed with visual grading regression (16). In the ordinal logistic model, patient and observer identity were treated as random effects and acquisition type (conventional or synthetic) as a fixed effect, and the calculations were performed in Stata 10.1 (Stata Corp LP, College Station, TX, USA) using $\operatorname{GLLAMM}(17,18)$. In cases in which the GLLAMM algorithm failed for numerical reasons, the analysis was instead performed with all independent variables treated as fixed effects in JMP 9.0 (SAS Inc., Cary, NC, USA). The agreement between observers concerning diagnosis was described with the unweighted kappa coefficient, computed with Stata 10.1. The frequency of correct diagnosis was compared between conventional and synthetic acquisition with binary logistic regression in JMP 9.0. 


\section{Results}

A typical example of quantitative MRI results is presented in Fig. 1. An axial slice is shown from one of the MS patients for the $R_{1}, R_{2}$, and $P D$ maps.
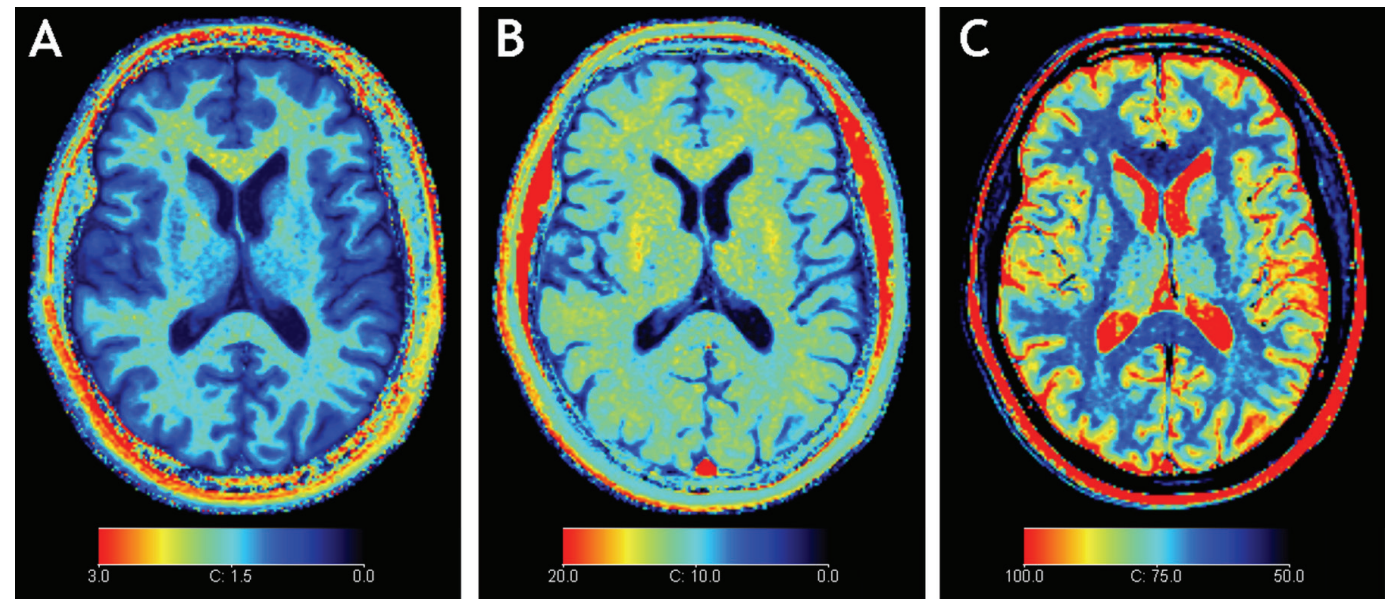

\section{Fig. 1 MR quantification maps of the brain}

A: The $R_{1}$ relaxation rate (scale $0-3 \mathrm{~s}^{-1}$ ), B: The $R_{2}$ relaxation rate (scale 0-20 $\mathrm{s}^{-}$

$\left.{ }^{1}\right)$ and $\boldsymbol{C}$ : The Proton Density (scale 50-100\% water) of an axial slice of the

brain of one of the MS patients.

Based on these quantitative measurements, synthetic images can be created, as exemplified in

Fig. 2, where synthetic T1-w, T2-w, and FLAIR images are compared with their

conventionally acquired counterparts. 

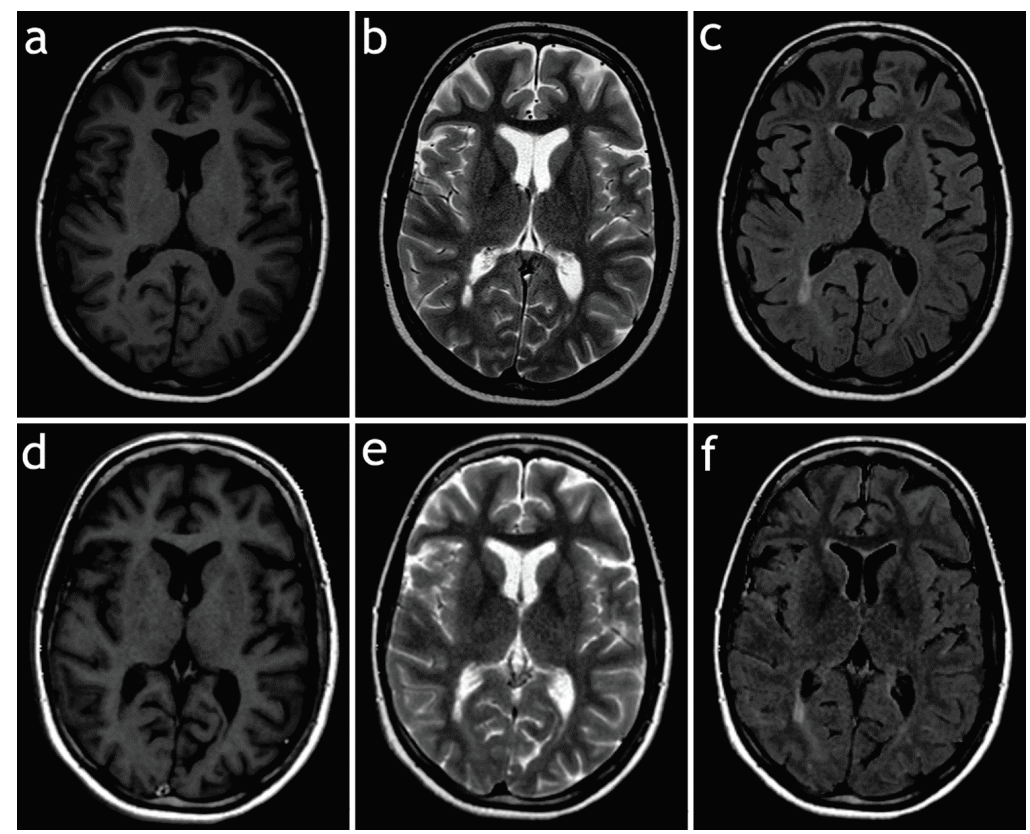

\section{Fig. 2 Example of conventional and synthetic images of the brain}

a: $T 1-w(T R / T E=350 / 15 \mathrm{~ms}), \boldsymbol{b}: T 2-w(T R / T E=4500 / 100 \mathrm{~ms}), \boldsymbol{c}:$ T2-FLAIR $(T R / T E / T I=$ $6000 / 120 / 2000 \mathrm{~ms})$. On the top row, standard conventional images are shown (a-c), on the bottom row, the corresponding synthetic images (d-f). All three synthetic images were created using the same quantification scan. The total scan time for the conventional images was 14.54 min, for the synthetic images $5.48 \mathrm{~min}$.

\section{Contrast and CNR measurements}

The results of image contrast and image CNR are presented in Fig. 3 with mean values and standard deviations. Linear regression showed that the synthetic T1-w images had $21 \%$ better contrast (with a $95 \%$ confidence interval (CI) of $15-28 \%$ ) but $7 \%$ lower CNR (CI: $1-14 \%$ ) than the conventional T1-w images. The synthetic T2-w images had 16\% better contrast (CI: 13-20\%) and 16\% lower CNR (CI: 14-19\%), FLAIR images had 19\% lower contrast (CI: 1324\%) and 38\% lower CNR (CI: 24-42\%).

If only grey matter-white matter and lesion-white matter contrast were considered, synthetic T1-w images had $32 \%$ better contrast and $2 \%$ higher CNR, synthetic T2-w images had 22\% 
better contrast and $20 \%$ higher CNR, and synthetic FLAIR images had $25 \%$ better contrast but $21 \%$ lower CNR.
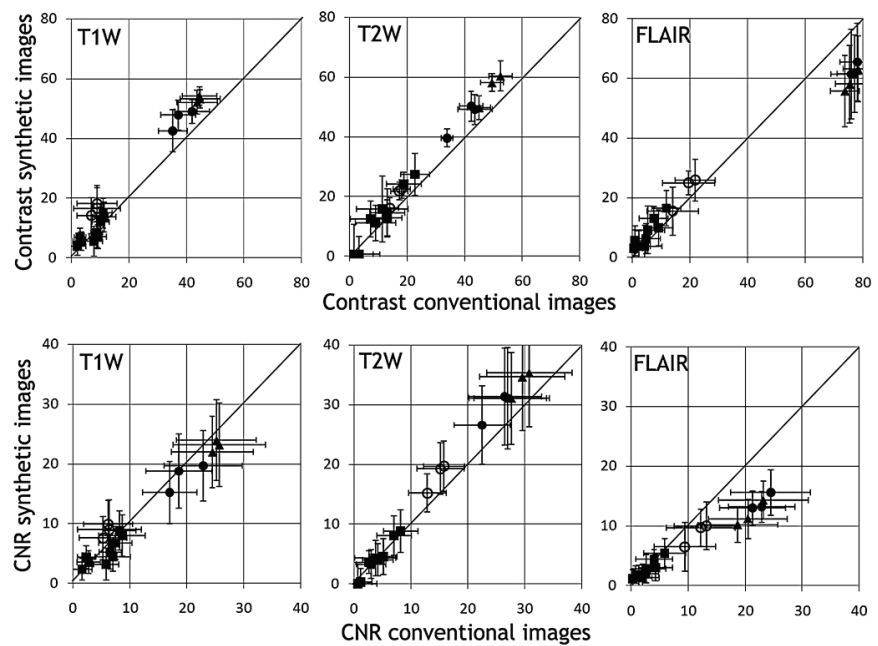

\section{Fig. 3 Correlation of contrast and contrast-to-noise ratio}

Contrast (top) and CNR (bottom) between synthetic and conventional T1-w, T2-w, and T2FLAIR images in \% units. The markers indicate the mean contrast and CNR value between each combination of the three grey matter structures with the three white matter structures (squares), each combination of CSF with the three grey matter structures (dots), each combination of CSF with the three white matter structures (triangles) and each combination of the MS lesions and the three white matter structures (circles). The error bars indicate one standard deviation. The diagonal lines indicate equality.

\section{Visual evaluation}

Scoring results for criteria 1-5 are plotted in Fig. 4. The differentiation between grey and white matter and the demarcation of sulci were found to be better in the conventional images $(P<0.0001$ for T1-w and T2-w, $P=0.046$ for FLAIR) compared with the synthetic ones. The demarcation of basal ganglia was better in the conventional T2w $(P<0.001)$ and FLAIR $(P=$ 0.003) sequences, whereas there was no significant difference in the $\mathrm{T} 1 \mathrm{-W}$ sequences. The perceived signal-to-noise level was lower in the synthetic images $(P<0.001)$. 


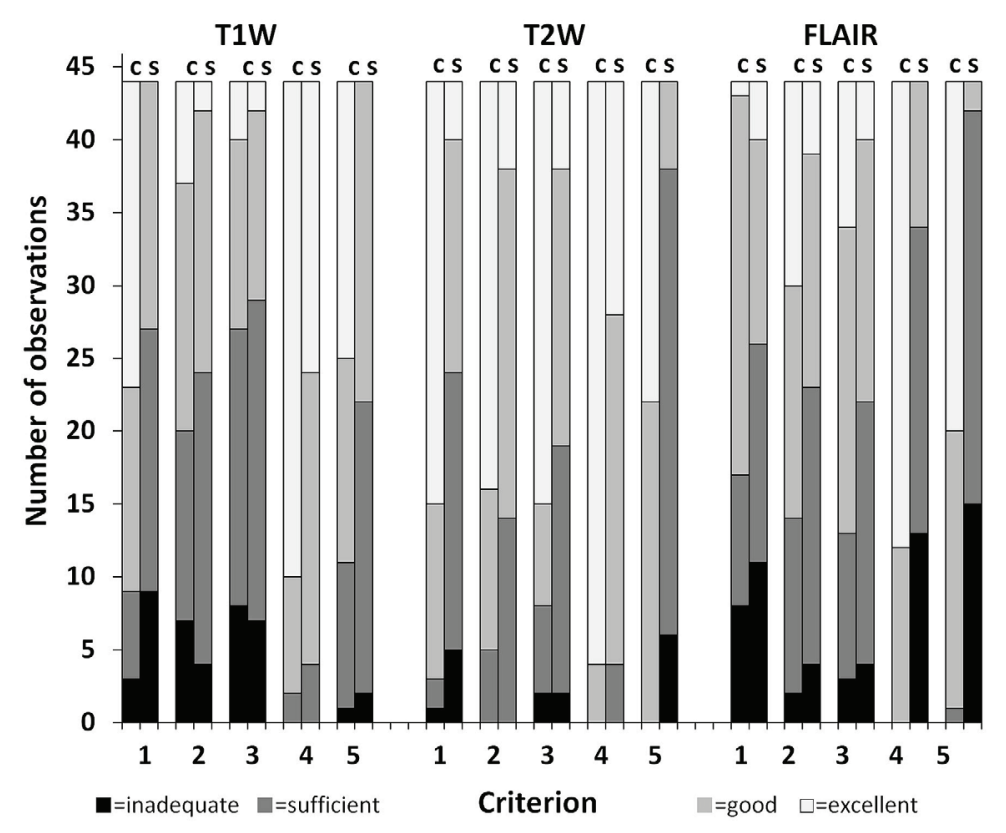

Fig. 4 Visual scoring diagram

Visual scoring diagram for criteria 1-5 for T1-w, T2-w, and T2-FLAIR images.

For each column the values for the conventional (c) images are given on the

left, for the synthetic images (s) on the right. Criterion 1: differentiation of grey-

white matter, criterion 2: demarcation of caudate nucleus head, criterion 3:

demarcation of the lentiform nucleus, criterion 4: demarcation of the sulci,

criterion 5: perceived level of signal to noise.

There was no significant difference in pulsation artifacts between the sequences. However, the synthetic T1-w and FLAIR images had more parallel imaging artifacts $(P<0.0001$ and $P=$ 0.004 respectively) and were more sensitive for other artifacts (e.g. motion) $(P<0.0001)$. The synthetic T2-w images had fewer of these other artifacts $(P=0.009)$.

Regarding the overall scoring for the eight quality criteria, $87 \%$ of the synthetic $\mathrm{T} 1-\mathrm{w}$ images were assessed to be of sufficient diagnostic quality or better, compared with $90 \%$ for the conventional T1-w images. The figures for T2-w images were $89 \%$ for the synthetic images 
compared with $95 \%$ for the conventional MRI. Eight-two percent of the synthetic FLAIR images were of diagnostic quality or better compared with $94 \%$ of the conventional FLAIR images.

Lesion conspicuity was assessed to be better in all of the synthetic T1-w images than in the conventional T1-w images. In the FLAIR and T2-w sequences, the conventional images had better lesion conspicuity ( $100 \%$ and $82 \%$, respectively).

There was no significant difference in the frequency of correct diagnosis between the synthetic and the conventional T2-w (77\% vs. $77 \%)$ and FLAIR (70\% vs. $73 \%)$ sequences. In the T1-w synthetic images, the frequency of a correct diagnosis was significantly higher (73\%) compared with conventional T1-w images $(64 \%, P=0.02)$. Inter-observer agreement regarding diagnosis was high, the greatest difference being seen in T1-w images, with kappa values of 0.85 for synthetic and 0.48 for conventional images. In the FLAIR sequences, kappa values were 0.86 for conventional images and 0.65 for synthetic images, and for T2-w images, 0.80 for conventional and 0.86 for synthetic images, respectively.

\section{Discussion}

In this study, the synthetic approach to MRI was compared with conventional MRI. Contrast and contrast-to-noise measurements showed a higher contrast in synthetic MR images than in conventional MR images, especially T1-w and T2-w images, but also a higher level of noise, resulting in comparable CNR values. Synthetic FLAIR images had lower image contrast as well as a higher noise level, resulting in a lower CNR than in conventional MR images. However, if only grey matter/white matter and lesion/white matter were considered, synthetic FLAIR images had a higher contrast. A higher noise level, especially in the areas with 
cerebrospinal fluid in the synthetic FLAIR images, can explain this difference. The reason why synthetic MR images exhibit higher contrast is unclear, but a possible explanation could be that conventional images are acquired with a longer echo train of typically $15-25$, in comparison to an echo train length of 6 for the quantification sequence. A high number of RF refocusing pulses may lead to a stronger magnetization transfer effect, resulting in a throughplane blurring of the images and hence loss of contrast.

Visual evaluation showed that synthetic images generally were inferior to conventional images with respect to delineation of grey and white matter and the sulci, but there was no significant difference in the T1-w images with regard to the basal ganglia. Even though the image quality was assessed as inferior for the synthetic images, $82-89 \%$ of the synthetic MRI datasets were of at least sufficient diagnostic quality compared with $90-95 \%$ of the conventional MRI datasets.

T1-w synthetic images had higher lesion conspicuity than conventional T1-w images, which could explain the higher number of correct diagnoses in synthetic T1-w images. There was no significant difference in frequency of correct diagnosis when comparing synthetic and conventional T2-w and FLAIR images, and in this respect synthetic MRI was comparable to conventional MRI for the diagnosis of demyelinating lesions and ischemia. In this study, with three alternative diagnoses, the MR-sequences were evaluated separately for quality criteria and probable diagnosis, whereas in clinical practice the diagnosis is based on the evaluation of several sequences.

Synthetic MRI has the potential to reduce the overall examination time by synthesis of multiple image contrasts based on a single, short quantification scan. Current development of new sequences and higher field strength has led to a focus on high-resolution imaging. For 
tissue characterization and diagnosis in neurodegenerative diseases, however, high resolution may not be absolutely necessary, and a rationale is required to balance the minimum scan time required for sufficient diagnostic information and the appeal to the eye. Hence, in this work the main focus was on the diagnostic quality of the synthetic MRI approach with a scan time $<6$ minutes in comparison with conventional clinical MRI protocols with a scan time between 10 and 15 minutes. The application of synthetic MRI in the clinical setting, however, would be facilitated by further improvements of the quantitative MRI sequence, especially with respect to reducing the current noise level. Implementation of the method at $3 \mathrm{~T}$ would provide the advantage of a higher signal-to-noise ratio. This could allow for thinner slices than in this study, which is required e.g. for detection of small MS-lesions.

In addition to the advantage of a shorter scan time, quantitative MRI has a great potential for automatic tissue characterization and volume estimation. Easy access to quantitative image information may provide radiologists with new tools for the diagnosis and monitoring of disease progress. Tissue relaxometry has been used in studies looking at different groups of patients, for example with epilepsy (19), MS (20, 21), and trauma (22), but could also be applied to patients with malignant brain tumors, where there is a great need to develop the diagnostic arsenal, e.g. for the differentiation of radiation necrosis and tumor progression (23). Further studies will evaluate this quantitative MRI method for volume measurements, lesion load, and atrophy development in MS patients, as well as tumor delineation and differentiation between tumor recurrence and radiation necrosis in patients with malignant gliomas. Synthetic MRI could also be used for the evaluation of ventricular size in patients with hydrocephalus. Another application is myelin measurements, which could be useful for the evaluation of MS-patients and for the assessment of the developing brain. Areas of potential body applications could be imaging and characterization of lesions in the breast and prostate. In this study, no quantitative measurements were made regarding the T1-, T2- and 
Pd-values, but previous work has dealt with tissue segmentation of the brain (11). Future work will analyze the effect of contrast agents on the quantitative values of brain tissue.

Limitations of our study are that our cohort included a small number of patients with a limited number of diseases, thus it would be desirable to study a larger patient population in the future.

The two conventional clinical MRI protocols differed in slice thickness and scan time. The MS protocol had thinner slices and a longer scan time than the standard brain protocol and the synthetic images, resulting in better delineation of anatomical structures and lesions.

In conclusion, synthetic MRI can potentially shorten the MRI examination time. Even though the image quality is perceived to be inferior, synthetic images agreed with the clinical diagnosis to the same extent as the conventional images and required half the scan time of the conventional images in this study.

\section{References}

(1) Riederer SJ, Lee JN, Farzaneh F et al. Magnetic resonance image synthesis. Clinical implementation. Acta Radiol Suppl 1986;369:466-8

(2) Riederer SJ, Suddarth SA, Bobman SA et al. Automated MR image synthesis: feasibility studies. Radiology 1984;153:203-6

(3) Bobman SA, Riederer SJ, Lee JN et al. Cerebral magnetic resonance image synthesis. Am J Neuroradiol 1985;6:265-9 
(4) Redpath TW, Smith FW, Hutchison JM. Magnetic resonance image synthesis from an interleaved saturation recovery/inversion recovery sequence. Br J Radiol 1988;61:619-24

(5) Gulani V, Schmitt P, Griswold MA. Towards a single-sequence neurologic magnetic resonance imaging examination: multiple-contrast images from an IR TrueFISP experiment. Invest Radiol 2004;39:767-4

(6) Deoni SC, Peters TM, Rutt BK. High-resolution T1 and T2 mapping of the brain in a clinically acceptable time with DESPOT1 and DESPOT2. Magn Reson Med 2005;53:237-41

(7) Maitra R, Riddles JJ. Synthetic magnetic resonance imaging revisited. IEEE Trans Med Imaging 2010;29:895-902

(8) Vymazal J, Righini A, Brooks RA et al. T1 and T2 in the brain of healthy subjects, patients with Parkinson disease, and patients with multiple system atrophy: relation to iron content. Radiology 1999;211:489-95

(9) Odrobina EE, Lam TY, Pun T et al. MR properties of excised neural tissue following experimentally induced demyelination. NMR Biomed 2005;18:277-84

(10) Larsson HB, Frederiksen J, Petersen J et al. Assessment of demyelination, edema, and gliosis by in vivo determination of $\mathrm{T} 1$ and $\mathrm{T} 2$ in the brain of patients with acute attack of multiple sclerosis. Magn Reson Med 1989;11:337-48

(11) West J, Warntjes JB, Lundberg P. Novel whole brain segmentation and volume estimation using quantitative MRI. Eur Radiol 2011;22:998-1007

(12) Lauterbur PC. Image formation by induced local interactions: examples employing nuclear magnetic resonance. Nature 1973;242:190-1 
(13) Warntjes JB, Leinhard OD, West J et al. Rapid magnetic resonance quantification on the brain: Optimization for clinical usage. Magn Reson Med 2008;60:320-9

(14) Borjesson S, Hakansson M, Bath M et al. A software tool for increased efficiency in observer performance studies in radiology. Radiat Prot Dosimetry 2005;114:45-52

(15) EUR 16262 EN. CEC European guidelines on quality criteria for computed tomography 1999. Luxembourg. http://www.drs.dk/guidelines/ct/quality/htmlindex.htm (Chapter1/Quality criteria/Brain, general)

(16) Smedby Ö, Fredrikson M. Visual grading regression: analysing data from visual grading experiments with regression models. Br J Radiol 2010;83:767-75

(17) Rabe-Hesketh S, Skrondal A, Pickles A. Reliable estimation of generalized linear mixed models using adaptive quadrature. The Stata Journal 2002;2:1-21

(18) Smedby Ö, Fredrikson M, De Geer J et al. Visual grading regression with random effects. SPIE Medical Imaging, San Diego, CA, United States, 2012.

(19) Bernasconi A, Bernasconi N, Caramanos Z et al. T2 relaxometry can lateralize mesial temporal lobe epilepsy in patients with normal MRI. Neuroimage 2000;12:739-46

(20) Hasan KM, Walimuni IS, Abid H et al. Multi-modal quantitative MRI investigation of brain tissue neurodegeneration in multiple sclerosis. J Magn Reson Imaging 2012;35:1300-11

(21) Larsson HB, Frederiksen J, Kjaer L et al. In vivo determination of T1 and T2 in the brain of patients with severe but stable multiple sclerosis. Magn Reson Med 1988;7:43-55

(22) Mamere AE, Saraiva LA, Matos AL et al. Evaluation of delayed neuronal and axonal damage secondary to moderate and severe traumatic brain injury using quantitative MR imaging techniques. Am J Neuroradiol 2009;30:947-52 
(23) Waldman AD. Magnetic resonance imaging of brain tumors- time to quantify. Discov Med 2010;9:7-12 